\title{
Analysis of the String Structure Near Break-up of A Slender Jet of An Upper Convected Maxwell Liquid
}

\author{
${ }^{1}$ A.Y. Gunawan $\&^{2}$ W.S. Budhi \\ ${ }^{1}$ Financial and Industrial Mathematics Group, FMIPA ITB, Indonesia \\ ${ }^{2}$ Geometry and Analysis Group, FMIPA ITB, Indonesia
}

\begin{abstract}
In this paper, we analytically study the string structure near the breakup of a slender jet of a viscoelastic liquid surrounded by air. The governing equations are derived from the conservation laws of mass and momentum, and the rheological equation of the jet. The rheological equation of the jet is assumed to satisfy an Upper Convected Maxwell (UCM) model. Introducing a stretch variable and then applying a transformation, we obtain a coupled system of nonlinear differential equations. Via these equations, we then show that the UCM jet does not break up in finite time, which physically means that it has sufficient time to exhibit the string structure before it breaks up due to the dominant surface force.
\end{abstract}

Keywords: Break-up; Slender jet; Stretch variable; UCM model.

\section{$1 \quad$ Introduction}

The break-up of fluid jets or threads by capillary instability is ubiquitous in engineering, science, and nature. For example, among others, an understanding of this phenomenon is essential to the technologies of ink-jet printing, the production of stable thin coatings, the morphology and stability of extruded polymer blends, the transportation of oil in pipeline, and tertiary oil recovery.

For Newtonian fluids, the asymptotics of jet break-up of Stokes flow was investigated by Papageorgiou [1] and presented in a more complete and elegant form by Eggers [2]. It was found that the asymptotic behaviour near the breakup is governed by similarity solution. The work of Pozrikidis [3] has shown that the pinching of liquid jet under the influence of surface tension can be described by similarity solutions of a simplified one-dimensional slender body model. The selfsimilar solution that describes finite time break-up for Newtonian fluid jet was discussed in detail by Renardy [4,5].

The work of Eggers [2] has been extended to non-Newtonian fluid, such as power law fluids by Renardy \&Renardy [6], and to various viscoelastic fluids, i.e. the fluids that exhibit elastic behaviour such as the memory effect as well as the fluid properties, such as a generalized PTT model by Renardy [7], generalized Newtonian fluid, K-BKZ model, and Dumbbell model by Renardy [8], and a Giesikus model by Renardy \& Losh [9]. They all showed that the 
similarity solution exists, which means that such fluids will break up in finite time. For instance, Renardy \& Losh [8] found that the similarity solution exists and reduces to the solution of Eggers [2] in the limit of infinite retardation time.

Beside it can break up in finite time, a viscoelastic fluid can give complicated rheological phenomena. For instance, a certain viscoelastic jet breaks up much more slowly than a Newtonian jet. Typically, it evolves into the so-called beadson-string structure, where two large drops are connected by a thin filament. The slow breakup process provides the viscoelastic jet sufficient time to exhibit this string structure. This structure continues to thin out as fluid from the ends drains into drops, but it disappears only after a very long time. A resistance of various viscoelastic jets to break-up (or the string structure) was studied theoretically by Renardy [10]. Li \& Fontelos [11] investigated the string structure and numerically showed that the elastic force is responsible for the drop migration and oscillation in the string structure. Oliveira \& McKinley [12] reexamined the dynamics of polymer solutions (viscoelastic fluids) using highspeed digital video microscopy. At long times, it was observed that the evolution of the viscoelastic thread deviates from self-similar exponential decay and the competition of elastic, capillary, and inertial forces leads to formation of a periodic array of beads connected by axially uniform ligaments. Clasen et al. [13] studied, both analytically and numerically, the beads-on-string structure of a liquid jet containing minute concentrations of a high-molecular-weight polymer. An addition of polymeric additives led to perform a stabilizing effect or the effect of delaying or even completely suppressing the break-up. To some extent, the asymptotics of the break-up for Non-Newtonian fluids, especially for viscoelastic fluids, is still open.

In this paper, we shall deal with the analysis of the string structure near the break-up of a viscoelastic liquid jet of an Upper Convective Maxwell liquid (UCM liquid). The elastic property of such fluid is described by the parameter, so called relaxation time. The relaxation time is a time lag between when the stress is released and when the material fully snaps back. Following the same line as in Renardy [4,10], we introduce a stretch variable and it leads us to the nonlinear system of partial differential equations. We then investigate the dynamics near the break-up. We find that the UCM jet does not break-up in finite time. This finding shows that the elasticity of the jet has a stabilizing effect. The present results may contribute to the explanation of the string structure that were observed in the breaking-up process of viscoelastic fluids. 


\section{Mathematical formulation}

We consider an axisymmetric liquid jet which is perturbed from a configuration of uniform fluid speed and uniform radius $a$, surrounded by air. The jet is assumed to behave as a viscoelastic material and we propose it as an Upper Convected Maxwell (UCM) liquid, which satisfies the rheological equation:

$$
\lambda\left(\frac{\partial \boldsymbol{T}}{\partial t}+(\boldsymbol{v} \cdot \nabla) \boldsymbol{T}-(\nabla \boldsymbol{v}) \boldsymbol{T}-\boldsymbol{T}(\nabla \boldsymbol{v})^{*}\right)+\boldsymbol{T}=\eta_{0}\left(\nabla \boldsymbol{v}+(\nabla \boldsymbol{v})^{*}\right),
$$

where $\boldsymbol{T}$ is the Stress tensor, $\boldsymbol{v}$ the velocity vector, $\lambda$ the viscoelastic relaxation time, $\nabla$ the gradient operator, ()* the transpose operator, and $\eta_{0}$ the zero-shearrate viscosity.

The kinematic of the motion is assumed to be one dimensional model. Here, we consider that the jet is thin, so called a slender jet, meaning that the axial velocity, the axial and the radial stress components do not vary much across the radius of the jet. Furthermore, we assume that the curvature in the azimuthal direction is much larger than the curvature in the axial direction. When the jet breaks up into spherical droplets, these assumptions are valid in the necks between the drops where the break-up occurs.

To formulate the governing equations, we shall follow the formulation derived by Renardy $[4,10]$. Let $r$ be the radial coordinate, $z$ be the axial coordinate, $u$ and $w$ be the radial and the axial components of the jet velocity, respectively, and $f(z, t)$ be the radius of the jet at point $z$ and time $t$. The equation of mass conservation leads to

$$
\frac{\partial f^{2}}{\partial t}+\frac{\partial\left(w f^{2}\right)}{\partial z}=0
$$

The incompressibility condition,

$$
\frac{\partial u}{\partial r}+\frac{u}{r}+\frac{\partial w}{\partial z}=0,
$$

for which at leading order it is satisfied by

$$
u=-\frac{r}{2} \frac{\partial w}{\partial z}
$$

This yields the velocity gradient

$$
\nabla \boldsymbol{v}=\left(\begin{array}{cc}
\frac{\partial w}{\partial z} & 0 \\
0 & -\frac{1}{2} \frac{\partial w}{\partial z}
\end{array}\right)
$$


Expression (5) will be used to calculate the equations of the stress components of (1) (see Equations (13) and (14) below).

Let $T_{z z}$ and $T_{r r}$ denote the axial and radial stress components of the stress tensor, respectively. In the interface between jet and air, we assume that there is a jump at the normal stress due to the present of surface tension. The jump is also proportional to the curvature of the jet surface. Physically, this jump cannot be negligible since we deal with the dynamic of the interface of two different fluids, i.e. the interface between jet and air, and it was also believed since many years that the surface tension is the source of instability (see, for instance, Eggers [2]). So, applying the Young-Laplace relation, the interfacial condition on the surface of the jet at leading order requires

$$
T_{r r}-p=-\frac{\sigma}{f(z, t)},
$$

where $p$ is the hydrostatic pressure which is assumed to be constant in a crosssection of the jet and $\sigma$ is the surface tension. In (6), we have applied a slender body approximation to the present jet.

Next, we shall derive the equation of the momentum. The force in the axial direction has components from the stress $\pi f^{2}\left(T_{z z}-p\right)$ and the surface tension $2 \pi f \sigma$. Neglecting the inertial forces, we obtain via Newton's law

$$
0=\frac{\partial}{\partial z}\left[\pi f^{2}\left(T_{z z}-p\right)+2 \pi f \sigma\right] .
$$

Substitution of (6) in (7) and then elimination of $\pi$ yield

$$
0=\frac{\partial}{\partial z}\left[f^{2}\left(T_{z z}-T_{r r}\right)+f \sigma\right] .
$$

Equations (1) and (8) are the governing equations for the problem at hand. To solve these equations, it is convenient to formulate the one-dimensional equations in a Lagrangian formulation (the undisturbed cylindrical configuration). Let $X$ be the position of a fluid particle in this reference configuration. Note that $z=z(X, t)$ is the actual position. From now on, we shall regard all variables as functions of $X$ and $t$. The stretch $s$, which describes the change in actual position $Z$ with respect to the reference position $X$, is defined by

$$
s=\frac{\partial z}{\partial X} .
$$

The equality of mixed partial derivative then yields (write $d s / d t=s_{t}$, for convenience) 


$$
s_{t}=w_{X} .
$$

Furthermore, from (10) we find, so called a kinematic relation,

$$
\frac{s_{t}}{s}=w_{z} .
$$

From Equations (2) and (11), we obtain a relation between the radius $f(z, t)$ of the cross-section of the jet after stretching and the stretch variable $s$ :

$$
f(z, t)=\frac{a}{\sqrt{s}} .
$$

The axial and the radial components of the stress tensor (1) in terms of the stretch $s$ are described by the ordinary differential equations:

$$
\begin{aligned}
& \lambda\left[\left(T_{z z}\right)_{t}-2 T_{z z} \frac{s_{t}}{s}\right]+T_{z z}=2 \eta_{0} \frac{s_{t}}{s}, \\
& \lambda\left[\left(T_{r r}\right)_{t}+T_{r r} \frac{s_{t}}{s}\right]+T_{r r}=-\eta_{0} \frac{s_{t}}{s} .
\end{aligned}
$$

We note that in the Lagrangian description, the time derivative represents the material time derivative and the velocity gradient has the form $s_{t} / s$. We next eliminate unnecessary constants by rescaling the variables. We set

$$
t=\frac{a \eta_{0}}{\sigma} \tilde{t}, T_{z Z}=\frac{\sigma}{a} \tilde{T}_{z Z}, T_{r r}=\frac{\sigma}{a} \tilde{T}_{r r} .
$$

In the sequel, we shall omit the tildes since confusion is not possible. Application of (12) into (8), substitution of (15) in (13) and (14), and then integration over $X$ lead to

$$
\begin{aligned}
& \frac{T_{z z}-T_{r r}}{s}+\frac{1}{\sqrt{s}}=c(t), \\
& W_{e}\left[\left(T_{z z}\right)_{t}-2 T_{z z} \frac{s_{t}}{s}\right]+T_{z z}=2 \frac{s_{t}}{s}, \\
& W_{e}\left[\left(T_{r r}\right)_{t}+T_{r r} \frac{s_{t}}{s}\right]+T_{r r}=-\frac{s_{t}}{s} .
\end{aligned}
$$

Here, $W_{e}=\lambda \sigma /\left(a \eta_{0}\right)$ is the Weissenberg number and $c(t)$ is undetermined integration constant, which is independent of $X$. The value of $c(t)$ is determined from the global constraint. Assuming that the perturbation of the jet is spatially periodic with period $L$, from the definition of $s$ in (9) we obtain

$$
\int_{0}^{L} s(X, t) d X=L .
$$

We can simplify Equations (16) -(18) if we set

$$
T_{z z}(X, t)=g(X, t) s^{2}(X, t)-W_{e}^{-1},
$$




$$
T_{r r}(X, t)=h(X, t) s^{-1}(X, t)-W_{e}^{-1} .
$$

The benefit of using (20) is to transform away the terms involving $s_{t} / s$ in (17) and (18). Equations (16)-(18) now become, after some modifications,

$$
\begin{aligned}
& g s^{3}-h+s^{3 / 2}=c(t) s^{2}, \\
& W_{e} g_{t}+g=\frac{s^{-2}}{W_{e}}, \\
& W_{e} h_{t}+h=\frac{s}{W_{e}}
\end{aligned}
$$

Up to now, we derived the equations for the dynamics of the jet radius in terms of variable $s$. In the next section, we shall analytically show that $s$ in (21)-(23) cannot blow up in finite time.

\section{$3 \quad$ No finite time break-up for the UCM jet}

In Renardy [10], one of the viscoelastic jets that he considered was the JohnsonSegalman fluid. For this model, he introduced three physical parameters: $\kappa, \mu$, and $a$, where $\kappa, \mu$, are positive constants, and $a$ is some number between -1 and +1 . Note that this $a$ differs from our $a$ in Section 2. He proved that for $1 / 2<a \leq 1$ the jet does not break up in finite time. This led to the conclusion that such fluid exhibits the string structure before it breaks up. Looking back to our model given by Equations (21)-(23), we can recognize that our model is a special case of his model if we take $a=1$ and $W_{e}=1 / \kappa=1 / \mu$. So, to be self-contained, we here apply the theorem derived by Renardy[10] and calculate explicitly all quantities regarding our model, such as the values of the lower and the upper bounds.

Theorem. Let $s(t)$ satisfies (21)-(23) subject to the constrain (19) and subject to positive initial values $g(X, 0)=g_{0}(X)$ and $h(X, 0)=h_{0}(X)$. For $t>0$, define

$$
m(t)=\min _{0 \leq X \leq L} s(t) \text { and } M(t)=\max _{0 \leq X \leq L} s(t) .
$$

Then $m(t)$ not approach zero nor does $M(t)$ approach infinity in finite time.

Proof. From (22) and (23) we obtain the following inequalities:

$$
\begin{aligned}
& a_{1}(t) \leq g \leq a_{1}(t)+a_{2}(t) \int_{0}^{t} m^{-2}(y) d y, \\
& b_{1}(t) \leq h \leq b_{1}(t)+b_{2}(t) \int_{0}^{t} M(y) d y,
\end{aligned}
$$


where $\quad a_{1}(t)=g_{0} e^{-t / w_{e}}, a_{2}(t)=\frac{1}{W_{e}^{2}}=b_{2}(t), b_{1}(t)=h_{0} e^{-t / w_{e}} . \quad$ Now, consider a point where $s=m$ or $s=M$, respectively. Equation (21) can be rearranged as

$$
c(t)=g s-h s^{-2}+s^{-1 / 2} .
$$

Application of (24) and (25) to (26) yields

$$
\begin{aligned}
& c(t) \geq a_{1}(t) M-b_{1}(t) M^{-2}-b_{2}(t) M^{-2} \int_{0}^{t} M(y) d y+M^{-1 / 2}, \\
& c(t) \leq a_{1}(t) m+a_{2}(t) m \int_{0}^{t} m^{-2}(y) d y-b_{1}(t) m^{-2}+m^{-1 / 2} .
\end{aligned}
$$

Integrating (21) over $[0, L]$, we find

$$
c(t) \geq-\frac{\int_{0}^{L} h d X}{\int_{0}^{L} s^{2} d X}
$$

Note that by Holder inequality

$$
\int_{0}^{L} s^{2} d X \geq \frac{\left(\int_{0}^{L} s d X\right)^{2}}{L}=L
$$

Moreover, by integrating (23) over $[0, L]$ we obtain an upper bound for the integral $h$. Hence, (30) yields a lower bound for $c(t)$ and then (28) yields a lower bound for $m$. Now, from (24) we find an upper bound for $g$. Using (21) at any point where $s \leq 1$ we now get an upper bound for $c(t)$. Application of this to (27) yields an upper bound for $M$.

Now, let

$$
N(t)=\max _{0 \leq X \leq L} g s-h s^{-2}+s^{-1 / 2} .
$$

Assume that $\bar{X}(t)$ is a point where the maximum of (31) is attained. Then we must have $s_{t}(\bar{X}(t), t) \leq 0$. Otherwise, $s_{t}(\bar{X}(t), t)>0$ will be contradictive with (19). Next we evaluate the derivative of $N(t)$ with respect to $t$, denoted by $N_{t}$, at the point $\bar{X}(t)$ :

$$
\begin{aligned}
N_{t} & =g_{t} s-h_{t} s^{-2}+s_{t}\left[g+2 h s^{-3}-\frac{s^{-\frac{3}{2}}}{2}\right] . \\
& =-\frac{1}{W_{e}}\left(N-s^{-\frac{1}{2}}\right)+s_{t}\left[g+2 h s^{-3}-\frac{s^{-\frac{3}{2}}}{2}\right] .
\end{aligned}
$$


Now, suppose $N(t)$ grows very large. Since (24) and (25) give an a priori bound for $g$, this is possible only $s$ becomes large. In that case, the term in square brackets in (32) is positive. So, in conclusion $N_{t}$ is negative if $N$ is too large. Hence, we obtain an upper bound for $N(t)$. Now, if $M(t)$ (see its definition in the Theorem) were becoming infinite in finite time, then it follows from (31) that $N(t)$ would also blow up. This is impossible according to what we have just addressed. Hence, the solutions cannot blow up in finite time.

\section{Conclusions}

We here derived the model to describe the string structure formation near the break-up of a viscoelastic liquid jet, based on the assumption that the jet behaves like a slender body. Taking an Upper Convected Maxwell (UCM) liquid as the rheological property of the jet, the governing equations led to a system of nonlinear partial differential equations. Via the stretch variable, we showed that the UCM jet does not break up in finite time. From a physical point of view, this elastic fluid may take longer time and exhibit a thin filament before breaking-up. This thin structure continues to thin out as fluid from the ends drains into drops, but it disappears only after a very long time. Here, the elasticity of the jet plays an important role as a delaying effect.

\section{Acknowledgement}

This research was supported by ITB-Research Grant no.174/K01.07/PL/2007. The Authors would like to thank the referees for their useful comments.

\section{References}

[1] Papageorgiou, D.T., On the breakup of viscous liquid threads, Physics of Fluids, 7, 1529-1544, 1995.

[2] Eggers, J., Nonlinear dynamics and breakup of free-surface flows, Reviews of Modern Physics, 3, 865-927, 1997.

[3] Pozrikidis, Capillary instability and breakup of a viscous thread, J. Engineering Mathematics, 36, 255-275, 1999.

[4] Renardy, M., Finite time breakup of viscous filaments, Zeitschrift Für Angewandte Mathematik und Physik (ZAMP), 52, 881-887, 2001.

[5] Renardy, M., A comment on self-similar breakup for inertialess Newtonian liquid jets, IMA J. Applied Mathematics, 70, 353-358, 2005.

[6] Renardy, M. \& Renardy, Y., Similarity solutions for breakup of jets of power law fluids, J. Non-Newtonian Fluid Mechanics, 122, 303-312, 2004.

[7] Renardy, M., Self-similar jet breakup for a generalized PTT model, J. Non-Newtonian Fluid Mechanics, 103, 261-269, 2002. 
[8] Renardy, M., Similarity solution for jet breakup for various models of viscoelastic fluids, J. Non-Newtonian Fluid Mechanics, 104, 65-74, 2002.

[9] Renardy, M. \& Losh, D., Similarity solutions for jet breakup in a Giesikus fluid with inertia, J. Non-Newtonian Fluid Mechanics, 106, $17-$ 27, 2002.

[10] Renardy, M., Some comments on the surface-tension driven break-up (or lack of it) of viscoelastic jets, J. Non-Newtonian Fluid Mechanics, 51, 97$107,1994$.

[11] Li, J, \& Fontelos, M.A., Drop dynamics on the beads-on-string structure for viscoelastic jets: A numerical study, Physics of Fluids, 15, 922-937, 2003.

[12] Oliveira, M.S.N, McKinley, G.H., Iterated stretching and multiple beadson-a-string phenomena in dilute solutions of highly extensible flexible polymers, Physics of Fluids, 17 (0717 04),1-4, 2005.

[13] Clasen, C., Eggers, J., Fontelos, M.A., Li, J., McKinley, G.H., The beadson-string structure of viscoelastic threads, Journal of Fluid Mechanics, 556, 283-308, 2006. 